\title{
Philosophiques
}

\section{La lecture foucaldienne de Descartes : ses présupposés et ses implications}

\section{Jean-Paul Margot}

Volume 11, numéro 1, avril 1984

URI : https://id.erudit.org/iderudit/203239ar

DOI : https://doi.org/10.7202/203239ar

Aller au sommaire du numéro

Éditeur(s)

Société de philosophie du Québec

ISSN

0316-2923 (imprimé)

1492-1391 (numérique)

Découvrir la revue

Citer cet article

Margot, J.-P. (1984). La lecture foucaldienne de Descartes : ses présupposés et ses implications. Philosophiques, 11(1), 3-39. https://doi.org/10.7202/203239ar
Résumé de l'article

Archéologie d'un même geste d'exclusion dont l'internement social de la folie et l'internement métaphysique de la déraison sont les effets, l'Histoire de la folie à l'âge classique décide de voir en Descartes le théoricien de cet acte violent de fondation de la raison occidentale. Après avoir exposé la polémique Foucault - Derrida, nous nous proposons, d'une part, d'identifier la " positivité » propre au discours foucaldien de la période " archéologique » et, d'autre part, de reprendre à notre compte la lecture de Descartes. Nous espérons alors montrer que le " coup de force " cartésien ne vise nullement à exclure la folie mais, au contraire, à forger rationnellement la fiction d'un monde qui serait sans ordre. Le monde sensible devient le lieu d'un simulacre, et ce qui le rend fascinant c'est la catastrophe imaginaire qu'il y a derrière, savoir la nature peut-être irrationnelle de l'univers. C'est de la nécessité de cette fiction que le sujet pensant va pouvoir émerger comme le lieu de la critique. 


\title{
ARTICLES
}

\section{LA LECTURE FOUCALDIENNE DE DESCARTES : SES PRÉSUPPOSÉS ET SES IMPLICATIONS}

\author{
par Jean-Paul Margot
}

RÉSUMÉ. Archéologie d'un même geste d'exclusion dont l'internement social de la folie et l'internement métaphysique de la déraison sont les effets, l'Histoire de la folie à l'âge classique décide de voir en Descartes le théoricien de cet acte violent de fondation de la raison occidentale. Après avoir exposé la polémique Foucault - Derrida, nous nous proposons, d'une part, d'identifier la «positivité " propre au discours foucaldien de la période " archéologique " et, d'autre part, de reprendre à notre compte la lecture de Descartes. Nous espérons alors montrer que le " coup de force " cartésien ne vise nullement à exclure la folie mais, au contraire, à forger rationnellement la fiction d'un monde qui serait sans ordre. Le monde sensible devient le lieu d'un simulacre, et ce qui le rend fascinant c'est la catastrophe imaginaire qu'il y a derrière, savoir la nature peut-être irrationnelle de l'univers. C'est de la nécessité de cette fiction que le sujet pensant va pouvoir émerger comme le lieu de la critique.

ABSTRACT. Being an archeology which, in a single excluding movement, produces the social confinement of madness and the metaphysical confinement of un-reason, The History of Madness in the Classical Age decides to see in Descartes the theoretician of this violent act of foundation of Western reason. After a presentation of the Foucault - Derrida polemic, the attempt is made, on the one hand, to identify the "positivity" proper to the Foucauldian discourse of the "archeological" period and, on the other hand, to re-read Descartes in a new light. One hopes to show that the Cartesian "revolution" in no way attempts to exclude madness but, on the contrary, to forge rationally the fiction of a world which would be without order. The sense world becomes the world of appearances, and what makes it fascinating is the imaginary catastrophe behind it, the knowledge of the 
perhaps irrational nature of the universe. It is by the necessity of this fiction that the thinking subject is going to be able to emerge as the place where the critique occurs.

La percée vers un langage d'où le sujet est exclu, la mise au jour d'une incompatibilité peut-être sans recours entre l'apparition du langage en son être et la conscience de soi en son identité, c'est aujourd'hui même une expérience qui s'annonce en des points bien différents de la culture : dans le seul geste d'écrire comme dans les tentatives pour formaliser le langage, dans l'étude des mythes et dans la psychanalyse, dans la recherche aussi de ce Logos qui forme le lieu de naissance de toute la raison occidentale. Voilà que nous nous trouvons devant une béance qui longtemps nous est demeurée invisible : l'être du langage n'apparaît pour lui-même que dans la disparition du sujet. M. Foucault.

$M$. Foucault ne consacre guère plus de quatre ou cinq pages à Descartes dans Folie et déraison. Cette courte référence peut, cependant, être considérée comme la clé explicative de son livre : "La folie dont la Renaissance vient de libérer les voix, mais dont elle a maîtrisé déjà la violence, l'âge classique va la réduire au silence par un étrange coup de force $"{ }^{1}$, celui de Descartes. La thèse de Foucault est posée sans ambages : il y a une synchronie temporelle et structurale entre le " grand renfermement " de la déraison et les Méditations métaphysiques de Descartes. Internement social et internement métaphysique de la folie sont les effets d'un même geste d'exclusion. L'occident, dès lors, va vivre sa raison dans le champ ouvert par la ségrégation de ce que Foucault appelle l'expérience tragique de la folie. Tout

1. M. Foucault, Histoire de la folie à l'âge classique, Paris, Tel, 1979, p. 56. Sauf indication contraire, toutes nos références à ce texte renvoient à cette édition. Il convient en effet d'indiquer que dans la première édition publiée chez Plon en 1961 apparaît une préface qui a été supprimée par Foucault dans l'édition Gallimard de 1972. Cette dernière, en revanche, est accompagnée de deux appendices : La folie, l'absence d'œuvre et Mon corps, ce papier, ce feu, qui ne figuraient pas dans l'édition de 1961 et qui ne figurent pas non plus dans l'édition de la collection Tel. Quant à la synchronie temporelle et structurale dont nous allons faire mention, voir E. Trías, Filosofia y carnaval, Barcelona, Anagrama, 1973. 
au contraire, selon J. Derrida, non seulement Descartes n'excluerait pas la folie dans l'économie du doute radical, mais il en installerait la possibilité menaçante au cœur même de l'intelligible et il ne permettrait à aucune connaissance déterminée de lui échapper en droit. En prenant appui sur la polémique Foucault-Derrida, nous nous proposons, d'une part, de montrer la " positivité " propre au discours foucaldien de Folie et déraison, son « a priori concret » et, d'autre part, de reprendre à notre compte, et de façon critique, la lecture qui est faite de Descartes. On n'aura donc d'autre dessein que celui de remettre en question la valeur discriminante que l'exemple de Descartes a dans Folie et déraison.

\section{1- L'ÉCONOMIE DU DOUTE CARTÉSIEN ET LA FOLIE.}

\subsection{La thèse de M. Foucault}

En moins de cent ans l'affrontement entre la « conscience critique » et " l'expérience tragique » de la folie disparaît pour laisser place au double internement de la folie. Rendue esclave par la raison, celle-ci est soumise aux règles éthiques du travail. C'est le passage de la Renaissance à la modernité, passage qui manifeste une nouvelle sensibilité sociale et culturelle au milieu du XVII ${ }^{\text {ème }}$ siècle en Europe. Les formes fantastiques de la déraison ne peuplent plus la demeure des hommes; la folie s'est installée dans la nuit silencieuse du monologue autoritaire de la ratio. La valeur exemplaire des Méditations ne réside pas dans le fait que Descartes serait une voix, une parmi d'autres, qui rend compte de cette opération de ségrégation, mais dans le fait que le philosophe de Touraine est le théoricien qui donne raison du " grand renfermement ». En effet, " dans l'économie du doute, il y a un déséquilibre fondamental entre folie d'une part, rêve et erreur de l'autre $»^{2}$. Si les sens et le rêve laissent un résidu de vérité, doute et folie s'excluent mutuellement puisque " c'est une impossibilité d'être fou, essentielle non à l'objet de la pensée, mais au sujet qui pense ", c'est-à-dire au sujet qui doute, qui assure la pensée contre la folie. Si le siècle de Montaigne voyait dans les menaces de la déraison la possibilité d'un compromis de la pensée avec la déraison, avec Descartes la raison

2. Histoire de la folie, p. 57. 
est exilée et exclue du discours de la déraison : "si l'bomme peut toujours être fou, la pensée, comme exercice de la souveraineté d'un sujet qui se met en devoir de percevoir le vrai, ne peut pas être insensée ${ }^{3}$. Se refusant de réanimer une vieille tradition, Foucault abandonne les interprètes classiques qui voient dans le rêve une folie généralisée et passe directement du doute des sens au doute du rêve.

\subsection{La critique de J. Derrida.}

Derrida voit dans l'argument du songe une exaspération hyperbolique de l'hypothèse de la folie, hypothèse que Descartes, loin d'exclure, adopterait face à un éventuel interlocuteur vulgaire : « je vais donc vous proposer une hypothèse qui vous paraîtra bien plus naturelle, qui ne vous dépaysera pas, parce qu'il s'agit d'une expérience plus commune, plus universelle aussi que celle de la folie : et c'est celle du sommeil et du rêve $"{ }^{4}$. L'essai de relecture "ingénue " l'amène à s'interroger sur la relation sémantique entre ce que, selon Foucault, Descartes dit et une certaine " structure historique ", c'est-à-dire une certaine totalité historique plein de sens. Afin d'atteindre le langage latent, il faut d'abord s'assurer du langage patent (signe). Or, c'est là que Foucault se trompe lorsqu'il affirme que Descartes " contourne l'éventualité du rêve ou de l'erreur ${ }^{5}$. Au contraire, " il faut bien comprendre ici que l'hypothèse du rêve est la radicalisation, ou si l'on préfere l'exagération hyperbolique de $l^{\prime}$ hypothèse où les sens pourraient parfois me tromper ${ }^{6}$. Ce que Foucault ne voit pas c'est que le résidu de l'hypothèse, ce qui résiste à l'hypothèse du rêve, conduit à des certitudes et à des vérités d'origine non sensible et non imaginative — « des choses fort simples et fort générales » - comme le sont les certitudes et les vérités mathématiques, et qu'il introduit une discontinuité dans les objets à partir d'une rupture avec les sens. Il n'y a donc pas une réduction continue, mais passage d'un ordre à un autre et la folie est un cas particulier de l'illusion

\footnotetext{
3. Ibid., p. 58.

4. J. Derrida, "Cogito et histoire de la folie ", in L'écriture et la différence, Paris, Le Seuil, 1967, p. 78.

5. M. Foucault, op. cit., p. 56.

6. J. Derrida, op. sit., p. 75
} 
sensible. L'économie de l'argumentation cartésienne dans la première méditation montre, en effet, que pour douter du résidu de vérité de la tromperie des sens il faut que « je me compare à ces insensés (insani) . . . qui assurent constamment qu'ils sont des rois, lorsqu'ils sont très pauvres ; qu'ils sont vêtus d'or et de pourpre, lorsqu'ils sont tout nus ; ou s'imaginent être des cruches, ou avoir un corps de verre ". Or, telle hypothèse a un résidu : " mais quoi ? ce sont des fous, et je ne serais pas moins extravagant, si je me réglais sur leurs exemples " ${ }^{7}$, et provoque l'étonnement du non-philosophe qui objecte que si l'on peut légitimement douter de la connaissance qui touche "les choses peu sensibles et fort éloignées ", il est impossible de douter de l'actualité du sujet qui est ici, assis auprès du feu, vêtu d'une robe de chambre, et d'autres choses de cette nature. De là que Descartes a recours à l'hypothèse du rêve qui, vu son caractère hyperbolique, lui permet d'être plus fou que le fou. Plus commune et plus universelle que celle de la folie, l'expérience du rêve finit par ruiner les fondements sensibles de la connaissance et par mettre à nu les fondements intellectuels de la certitude. Le niveau patent du texte cartésien oblige à réintroduire dans son lieu et dans sa fonction l'hypothèse de la folie et à voir qu'elle ne représente pas la pensée définitive de Descartes. Elle n'est qu'un maillon qui, parmi les raisons naturelles de douter, facilite le passage à un argument plus radical. L'hypothèse de la folie s'inscrit à l'intérieur du mouvement d'exacerbation des raisons que nous avons pour douter des sens et nous conduit à l'intelligible, c'est-à-dire à la disqualification épistémologique du sensible. L'hypothèse de « l'extravagance » n'est donc pas un exemple révélateur, alors que l'est celle du malin génie où est convoquée « la possibilité d'une folie totale, d'un affolement total " ${ }^{8}$. Et le cogito est le moment où l'on échappe à la folie parce que "l'acte du cogito vaut même si je suis fou, même si ma pensée est folle de part en part " ${ }^{9}$. "L'audace folle " du cogito consiste en ce que fou ou non, cogito, sum. Dans la mesure où il échappe

7. R. Descartes, Première méditation. Nous utiliserons l'édition de F. Alquié des Oeuvres philosophiques de Descartes, Paris, Garnier, 3 volumes, 1967. "Sed amentes sunt isti, nec minus ipse demens viderer, si quod ab iis exemplum ad me transferrem (si je m'appliquais quelque exemple venant d'eux) ».

8. 'J. Derrida, op. cit., p. 81 .

9. Ibid., p. 85. 
à l'alternative d'une folie et d'une raison déterminées, le cogito apparaît comme le point à partir duquel se donne l'histoire des relations entre le sens et la déraison. Mais, dans la mesure où il est le fondement de la certitude, il est aussi le "point où s'enracine le projet de penser la totalité en lui échappant $"{ }^{10}$ puisque, seul à pouvoir résister à l'hypothèse de la folie de l'univers, il est également le seul qui permette de projeter un sens dans l'histoire : « c'est pourquoi, en cet excès du possible, du droit et du sens sur le réel, le fait et l'étant, ce projet est fou et reconnaît la folie comme sa liberté et sa propre possibilité ${ }^{11}$.

\subsection{La réponse de Foucault.}

C'est un fait indéniable que dans le cheminement du doute, Descartes donne un privilège au rêve sur la folie. Mais il ne dit jamais que le rêve est " plus commun, plus universel aussi que la folie ». Il ne dit pas non plus que la folie est un état momentané et particulier. L'erreur de Derrida consiste à confondre le caractère démonstratif du rêve comme exemple d'extravagance et son caractère accessible comme exercice que la fréquence du rêve assure. Il ne voit pas que Descartes donne une plus grande importance au caractère accessible qu'au caractère démonstratif. Le rêve, par conséquent, n'est pas l'exaspération hyperbolique de l'hypothèse de la folie. S'il perturbe le sujet qui pense, s'il le stupéfie (stupor), c'est parce qu'il efface les frontières qui délimitent le rêve et la veille. Il ne l'empêche toutefois pas de méditer, c'est-à-dire d'exercer sa pensée puisque, tout en poussant jusqu'à l'extrême l'hypothèse du rêve, le sujet est capable de voir avec clarté certaines choses. Le rêve n'est donc pas « universel » mais commun au sujet qui médite et qui « peut poursuivre de façon sûre le cheminement de son doute ". De plus, la comparaison des paragraphes que Descartes consacre à la folie et au rêve met en évidence l'exclusion de la folie et sa nonprologation dans le rêve. Il y a un "système d'opposition complexe qui les sous-tend », et il est " difficile de n'y pas reconnaître deux exercices à la fois parallèles et différents : l'exercice du demens et celui du dormiens ${ }^{12}$. Le fait que l'on vérifie immé-

10. Ibtd., p. $86-87$

11. Ibid., p. 87.

12. M. Foucault, Histoire de la folie (1972), "Mon corps, ce papier, ce feu ", p. 589. 
diatement la non-folie et que l'on prouve de façon discursive le rêve montre la discontinuité radicale qui existe entre l'expérience de la folie et l'hypothèse du rêve en tant qu'étape du doute. L'analyse des termes latins "insani » (insensés), " amentes » (fous) et « demens » (extravagant) montre aussi que « Descartes joue sur l'écart entre deux types de déterminations de la folie (les unes médicales et les autres juridiques) ${ }^{13}$. Selon le lieu qu'ils occupent et la fonction qu'ils remplissent dans le texte, ils sont soit un terme médical caractérisant, insanus, soit des termes juridiques disqualifiants, amens et demens. Si le premier est un signe, les deux autres disqualifient le sujet pensant. Le " insanus" est un objet médical que la meditatio caractérise. Mais la méditatio contourne le danger de la folie parce que la démence rend le sujet incapable de penser. Au contraire, si je feins de rêver, alors, tout dormiens que je suis, je pourrai continuer à méditer et à raisonner. Si la folie est exclue par le sujet qui pense, qui doute ${ }^{14}$, ce n'est pas pour le contenu de ses extravagances, mais pour que le sujet puisse se qualifier comme sujet doutant.

La double lecture que requièrent les Méditations invalide l'interprétation classique selon laquelle la folie apparaît avec l'bypothèse du Dieu trompeur. Quelle est cette double lecture ? : " un ensemble de propositions formant système, que chaque lecteur doit parcourir s'il veut en éprouver la vérité ; et un ensemble de modifications formant exercice, que chaque lecteur doit effectuer, par lesquelles chaque lecteur doit être affecté, s'il veut être à son tour le sujet énonçant, pour son propre compte, cette vérité ${ }^{15}$. Bien qu'il reconnaisse que la forme discursive "système " se croise avec la forme discursive " ascèse ", Foucault donne un privilège au système sur l'exercice parce qu'il croit ou décide - qu'il en est ainsi dans l'économie de la première méditation. Contre la lecture historico-transcendantale des traces derridiennes, Foucault affirme que le sujet ne crée pas le discours, que le discours crée ses objets et que l'on a affaire à un

\footnotetext{
13. Ibid., p. 591.

14. Une chose qui pense est pour Descartes « une chose qui doute, qui conçoit, qui affirme, qui nie, qui veut, qui ne veut pas, qui imagine aussi, et qui sent ", Méditation seconde. La pensée est l' " attribut principal " de la substance pensante, et c'est à elle que se réferent les modes de la pensée ; voir Les principes de la philosophie, I, 53.

15. M. Foucault, op. cit., p. 594.
} 
sujet dispersé. Face au système qui le précède et le détermine ${ }^{16}$, le sujet perd sa souveraineté et son pouvoir décisoire d'exclusion. Mais ceci ne serait tout au plus qu'une hypothèse ou un cadre théorique qu'il faudrait vérifier. De là le choix du doute cartésien et la longue réponse à Derrida. En effet, au niveau de la « discursivité systématique ", la première méditation n'est autre qu'un ensemble d'énoncés produits dans l'espace et dans le temps et qui ont, en tant qu'événements, une matérialité irréductible. Ils constituent, en leur niveau démonstratif, une série d'événements liés entre eux selon un système réglé de différences et de dispersion. Or là, le sujet ne joue aucun rôle hégémonique. La démonstration ne l'engage à rien puisque, comme les autres événements, il est soumis à la rigueur démonstrative-logique de l'enchaînement des énoncés : c'est un invariant. En revanche, au niveau de la " discursivité ascétique ", la méditation compromet le sujet. La production de nouveaux énoncés provoque des modifications dans la structure du sujet connaissant et énonçant. Celui-ci décide, choisit, sélectionne et, surtout, valorise contre l'ordre du système la position d'un sujet qui, s'il veut se maintenir, doit être un sujet mobile. Le mouvement de la méditation provoque des déplacements textuels qui entraînent avec eux des modifications du sujet énonçant : « dans la méditation, le sujet est sans cesse altéré par son propre mouvement ; son discours suscite des effets à l'intérieur desquels il est pris ; il l'expose à des risques, le fait passer par des épreuves ou des tentations, produit en lui des états, et lui confere un statut ou une qualification dont il n'était point détenteur au moment initial ${ }^{17}$. Parce qu'il n'a pas su - ou mieux, parce qu'il n'a pas voulu - reconnaître ces différences discursives, Derrida s'est trompé ; les passages sur la folie et sur le rêve peuvent seulement se comprendre depuis cette double lecture des Méditations. Car s'il existe une zone de lumière qui résiste à la nuit du doute des sens, si Descartes argumente qu'il y a des choses sensibles dont " on ne peut pas raisonnablement douter ", c'est parce que le sujet ne peut pas effectuer réellement, dans l'exercice qui le modifie lui-même, un doute si général. Que la folie ne soit pas une étape du doute veut donc dire que Descartes décide de rompre

16. Notons ici le spinozisme de Foucault.

17. M. Foucault, op. cit., p. 593-594. 
avec la logique du système pour passer au niveau de la " méditation ", c'est-à-dire de l' « exercice ». Et c'est en effet d'une décision éthique dont il s'agit ${ }^{18}$ puisque recourir à la stricte nécessité de la démonstration (système) disqualifierait le sujet. Recourir au système rendrait impossible l'exercice méditatif : Le sujet ne serait plus raisonnable. Pour éviter que cela n'arrive, il faut donc passer de la reconnaissance de celui qui sait qu' il doit douter à la nécessité d'appliquer — avec des restrictions - le doute, si le sujet ne veut pas disparaître dans la déraison. Mais résister à l'universalité de la tromperie sensorielle, c'est aussi se refuser à interroger le système d'actualité qui caractérise ce moment-ci de ma méditation, savoir « que je sois ici, assis auprès du feu, vêtu d'une robe de chambre, ayant ce papier entre les mains, et autres choses de cette nature $"{ }^{19}$. Car, si je doutais de mon actualité, je serais fou et je me disqualifierais comme sujet raisonnable, c'est-à-dire comme sujet capable de méditer. Il faut donc passer du système à l'ascèse. En revanche, l'exemple du rêve, même s'il rend l'actualité du sujet non moins douteuse que la folie, fait partie des virtualités du sujet : je suis un homme qui a coutume de dormir et de rêver et qui a des souvenirs. En d'autres termes, si la folie exclut la possibilité de méditer, le rêve maintient le sujet au sein de l'exercice de la méditation. Cette qualification du sujet enfin acquise (Age somniemus), la discursivité systématique va donc pouvoir croiser à nouveau le discours de l'exercice, prendre le dessus, mettre en examen les vérités intelligibles, jusqu'à ce qu'un nouveau moment ascétique constitue le sujet méditant comme menacé d'erreur universelle par le «grand trompeur ». Mais l'exclusion préalable

18. M. Foucault écrit, op. cit., p. 157 : « À l'âge classique, la raison prend naissance dans l'espace de l'éthique. Et c'est sans doute ce qui donne à la reconnaissance de la folie à cette époque - ou, comme on voudra, à sa non-reconnaissance - son style particulier. Toute folie cache une option, comme toute raison un choix librement accompli. Ceci se laisse deviner dans l'impératif insistant du doute cartésien ; mais le choix lui-même, ce mouvement constitutif de la raison, dans lequel la déraison est librement exclue, se dévoile tout au long dans la réflexion de Spinoza, et les efforts inachevés de la Réforme de l'entendement ". S'il est vrai que " la raison prend naissance dans l'espace de l'éthique ", nous nous refusons de suivre Foucault dans sa lecture de Descartes et de Spinoza. Nous aurons l'occasion de montrer que la séparation - si séparation il y a - entre raison et folie est la sédimentation archaïque de la pensée occidentale. Il faudra également montrer que la raison foucaldienne prend elle aussi naissance dans le champ de l'éthique. N'est-ce pas Foucault qui a baptisé du nom de déctsion la dissension entre la raison et son autre ? Et n'est-ce pas une valorisation éthique celle qui consiste à choisir l'âge classique, et cela même si L'archéologie du savoir la justifie par des raisons méthodologiques?

19. Descartes, Première méditation, vol. II, p. 405. 
de la folie fait que la fiction soit maintenant celle d'un sujet raisonnable. Cette hypothèse n'est donc pas le moment de la " folie totale ", de "l'affolement total » car, contrairement au fou qui croit être roi lorsqu'il est mendiant, "l'hypothèse du malin génie me permet de ne pas croire que ce corps et ces mains existent. Si le malin génie reprend les puissances de la folie, c'est après que l'exercice de la méditation a exclu le risque d'être fou ${ }^{20}$. S'il n'y a pas dans l'économie du doute une rupture au niveau de l'hypothèse du Dieu trompeur, c'est parce que la rupture s'est opérée auparavant, lorsque Descartes décida de choisir la pensée contre l'extravagance.

\section{2- LA POSITIVITÉ DU DISCOURS FOUCALDIEN.}

\subsection{Le problème du sens et la notion du sujet.}

La réponse de Foucault va au-delà d'un simple intérêt exégétique. De fait, si Derrida ne tient pas ses promesses d'interroger le texte cartésien à son niveau patent, ce n'est pas par inattention mais par "système ". Aux traces derridiennes, aux marques pour une lecture, aux voix derrière les textes et à l'assignation de l'originaire comme dit et non dit dans le texte, Foucault oppose les pratiques discursives, les événements, les modes d'implication du sujet dans les discours et le champ des transformations dans lequel s'effectuent les pratiques discursives. La publication en 1969 de l'Archéologie du savoir lui permet de dénoncer la "textualisation " derridienne comme "une petite pédagogie historiquement bien déterminée ${ }^{21}$. Il n'y aurait rien hors du texte, mais le texte recouvrirait des non-dits qui le soustendent et qui sont le lieu du sens, le lieu où règne « la réserve de l'origine ». Ce qui, par conséquent, est en jeu dans la différence entre la trace derridienne et l'archéologie foucaldienne est une théorie de la lecture et de l'écriture, ainsi qu'une réflexion sur la notion de sujet ${ }^{22}$. Lire n'est pas, pour Foucault, dé-construire mais construire le texte ; lire n'est pas placer le texte sous le double signe de la totalité et de la pléthore, mais prendre le

20. M. Foucault, op. cit., p. 601.

21. lbid., p. 602.

22. Voir D. Giovannangeli, Écriture et répétition, Paris, 10/18, 1979, p. 161 et suiv. 
texte au niveau des seuls ensembles signifiants qui ont été énoncés et des formations discursives. Or, "l'analyse des énoncés s'effectue sans référence à un cogito ${ }^{23}$. Ce qui est mis en question, c'est le thème général de la " connaissance ", savoir la continuité de la science et de l'expérience, le thème d'une « activité constituante qui assurerait . . . l'unité entre une science définie par un système de réquisits formels et un monde défini comme l'horizon de toutes les expériences possibles », le thème d'un sujet, et surtout « le grand thème historico-transcendental avec son telos qui permet que la vérité se fasse jour et révèle dans l'histoire ce que l'origine avait occulté ${ }^{24}$. Afin de prendre toute la mesure de la critique de Foucault à Derrida, il faut comprendre que " « n'importe qui parle », mais ce qu'il dit, il ne le dit pas de n'importe où. Il est pris nécessairement dans le jeu d'une extériorité ${ }^{25}$.

Dans cette perspective le silence qui est tombé sur Maladie mentale et personnalité n'est pas accidentel, et il convient de voir pourquoi Foucault imposa ce silence et comment celui-ci explique et compromet toute la réflexion postérieure de Foucault. Rappelons que le dessein de ce livre était de « montrer que la racine de la pathologie mentale ne doit pas être cherchée dans une quelconque " métapathologie », mais dans un certain rapport, historiquement situé, de l'homme à l'homme fou et à l'homme vrai ${ }^{26}$. Cette revendication de l'homme peut certes paraître paradoxale, voire contradictoire, pour un des prédicateurs de la mort de l'homme. La formation culturelle de Foucault éclaire

23. M. Foucault, L'archéologie du savoir, Paris, Gallimard, 1969, p. 161. "Il ne faut plus situer les énoncés par rapport à une subjectivité souveraine, mais reconnaître dans les différentes formes de la subjectivité parlante des effets propres au champ énonciatif », ibld, p. 160.

24. M. Foucault, "Réponse au cercle d'épistémologie ", in Cabiers pour l'analyse, no 9, Paris, été 1968 , p. 39-40.

25. M. Foucault, L'archéologie du savoir, p. 161.

26. M. Foucault, Maladie mentale et psychologie, Paris, P.U.F., 1966, p. 2. La première édition est de 1954 et apparaît sous le titre de Maladie mentale et personnalité : Dans l'édition de 1966 Foucault a substitué à la deuxième partie du livre un matériel entièrement nouveau, modifiant ainsi et la thèse du livre, et la base conceptuelle de la psychiatrie. A. Sheridan écrit : "The real heroes are not the sober, white-coated scientists patiently pushing back the clouds of ignorance and painfully revealing, little by little, the true nature of madness, but rather those literary "madmen" who, repudiating the language of reason, crossed over into the territory of "unreason" and, in a language beyond and prior to both, testified to an experience that lay, not beyond the boundary of true humanity, but at its heart" in, M. Foucault. The will to truth, New York, Tavistock publications, 1980, p. 7. 
cependant cette situation. Ainsi que beaucoup d'autres intellectuels de sa génération, Foucault s'est formé " à l'école de la phénoménologie, dans l'analyse des significations immanentes au vécu, des significations implicites dans la perception et dans l'histoire. De plus, affirme-t-il, je m'intéressais à la relation qui pouvait exister entre l'existence individuelle et l'ensemble des structures et des conditions historiques dans lesquelles apparaît l'existence individuelle; le problème des relations entre sens et histoire, et même entre méthode phénoménologique et méthode marxiste ${ }^{27}$. Entre 1950 et 1955 , Foucault souffre une " espèce de conversion » qui l'amène à s'occuper des conditions formelles qui déterminent l'apparition de la signification. À partir de 1955 cette préoccupation se transforme en axe fondamental de sa réflexion, pour le moins jusqu'à certains déplacements thématisés dans l'ordre du discours. Fidèle à sa formation phénoménologique (l'idée husserlienne selon laquelle il y a du sens), Foucault peut affirmer que même lorsqu'elle est réduite au silence et exclue, la folie a une valeur de langage et que son contenu prend un sens à partir de ce qui la rejette et la dénonce comme folie. Mais l'analyse des conditions sous lesquelles apparaît la maladie mentale nous conduit maintenant vers la problématique propre à l'Histoire de la folie à l'âge classique. Analyser ces conditions, c'est commencer à interroger les corpus scientifiques qui surgissent vers la fin du XVIIrème siècle et qui transforment petit à petit en un objet scientifique les significations immédiatement vécues par une société déterminée. Or, encore influencé par le marxisme de son époque, Foucault découvre ces conditions d'apparition de la maladie mentale dans l'aliénation sociale. Afin de définir la maladie mentale, il faut partir de ses conditions réelles - historiques et humaines. Maladie mentale et personnalité s'explique donc par le cadre culturel dans lequel se meut Foucault en 1954. S'il parle d'individu, de personnalité, c'est parce qu'il dépend de Husserl et d'une certaine prétention universaliste : « . . . la méthode phénoménologique, en effet, veut rendre compte de tout, aussi bien du cogito que de ce qui est antérieur à la réflexion, de ce qui « est déjà » lorsque commence l'activité du cogito, et en ce sens elle est une méthode totalisante ${ }^{28}$.

27. Conversazioni con Claude Lévi-Strauss, Micbel Foucault, Jacques Lacan, a cura di Paolo Caruso, Milano, U. Mursia \& C., 1969, p. 94.

28. Ibid., p. 111-112. 
Qui plus est, la préoccupation marxiste pour la totalité, pour le réel, pour l'homme réel, envahit la réflexion de Foucault.

\subsection{Expérience tragique et conscience critique.}

Laissant de côté la phénoménologie, L'Histoire de la folie à l'âge classique aborde le problème de la folie à partir d'une perspective apparemment plus "marxiste ${ }^{29}$. De fait, publié en 1961, ce deuxième livre de Foucault fut initialement pensé comme une contribution à ce qui occupait les intellectuels de gauche en France, savoir le rapport idéologie-science. Cette dette une fois signalée, il n'est pas difficile de voir dans la lecture foucaldienne de l'économie cartésienne du doute un échantillon d'une importance singulière de l'exclusion de l'idéologie (folie) par la science (raison). On doit pour le moins reconnaître que s'il est vrai que Foucault pose ce problème en des termes relativement nouveaux, il se meut à l'intérieur d'une pensée binaire qui détermine toute son " histoire " de la folie comme une confrontation des dialectiques de l'histoire avec les structures immobiles du tragique ${ }^{30}$. Foucault, certes, ne place pas la notion de sujet sur la scène rationaliste de l'opposition entre la vérité et l'erreur. Dans la polémique qu'il engage avec Althusser dans l'archéologie du savoir, il nous dit que la scène rationaliste a été remplacée par le " champ de savoir " dans lequel la science se localise : « la question de l'idéologie posée à la science, ce n'est pas la question des situations ou des pratiques qu'elle reflète d'une façon plus ou moins consciente ; ce n'est pas la question non plus de son utilisation éventuelle ou de tous les mésusages qu'on peut en faire ; c'est la question de son existence comme pratique discursive et de son fonctionnement parmi d'autres pra-

29. Ces guillemets devraient être l'objet d'une étude à part sur les relations que Foucault maintient tout au long de son ouvre avec le/s marxisme/s. Ne pas faire mention de Marx n'est pas une garantie que l'on n'est pas marxiste. Mais faire mention de Marx n'est pas une garantie que l'on est marxiste. En réalité, le fait que nous placions le texte de Foucault à l'intérieur du débat idéologie-science est un indice suffisant. Il suffit de rappeler que L. Althusser publia " les manifestes philosophiques " en 1960, "Sur le jeune Marx " en 1961, "Contradiction et surdétermination " en 1962 pour se faire une idée du climat théorique dans lequel apparaît l'Histoire de la folie. Pensons également aux relations de Foucault avec J. Hyppolite, qui est un de ceux qui introduisirent Hegel dans l'université française. En 1961 Marx et Hegel sont à l'ordre du jour, comme le laisse entrevoir la préface de la première édition de l'Histoire de la folie.

30. M. Foucault, Histoire de la folie (1961), préface. 
tiques $»^{31}$. Toutefois, et même s'il est vrai que Foucault n'a jamais été le positiviste qu'on l'a accusé d'être ${ }^{32}$, on ne peut pas nier que le fait de privilégier le "système " sur "l'exercice " n'est pas moins rationaliste que le fait d'exclure l'idéologie à partir de la science. En réalité, si comme dit G. Canguilhem, " ce fut à Descartes à qui l'auteur réel de l'bistoire de la folie confia la tâche de nous présenter l'idée de la folie à l'âge classique $»^{33}$, c'est, croyons-nous, parce qu'il garde la nostalgie d'une " expérience tragique " de la folie que la ratio cartésienne enferma métaphysiquement. C'est ainsi que sa lecture devient elle aussi sujette au doute, non pas tant pour l'exégèse du texte cartésien, mais pour les présupposés de sa lecture. Nous avons la sensation que si Foucault fait exclure la folie par Descartes, ce n'est pas parce que l'économie de la première méditation le lui permet mais parce qu'il faut que cela soit ainsi afin de pouvoir écrire que « cette expérience tragique subsiste dans les nuits de la pensée et des rêves " et que l'internement de la folie ne fut pas une destruction mais seulement une "occultation ${ }^{34}$. Nous avons la sensation qu'il y a une certaine ingénuité à penser que pour valoriser les œuvres de Sade et de Goya, de Nietzsche, Van Gogh et Artaud il est nécessaire de montrer comment un "étrange coup de force » a été incapable de taire ad eternam l'expérience tragique de la folie $^{35}$. En d'autres termes, nous croyons que si Foucault privilégie "l'expérience " cartésienne ce n'est sans doute pas pour exclure la folie (idéologie) à partir de la raison (science), mais pour exclure la raison et l'Homme à partir d'une expérience tragique ineffable de la folie. L'opération est séductrice ; mais dans l'ordre des exclusions personne n'a de privilège. La réponse de Foucault à Derrida s'inscrit dans la distance qui existe entre la mise à nu de l'expérience tragique de la folie et de ses vestiges qui

31. M. Foucault, L'archéologie du savoir, p. 242.

32. Sur le posıtivisme de Foucault, voir S. Le Bon : "Un positiviste désespéré : Michel Foucault ", Les temps modernes, no 248, janvier 1967, p. 1299-1319.

33. "Mort de l'bomme ou épuisement du cogito?". Critique, no 242, juillet 1967, p. 599.

34. M. Foucault, Histoire de la folie, p. 39.

35. Si l'on en croit l'auteur de L'archéologie du savoir, la méthode archéologique ne prétend pas démontrer le réel, mais le montrer. Ce qui intéresserait Foucault ne serait pas la normativité de l'histoire des sciences, normativité facilitée par un certain réductıonnisme épistémologique, (Bachelard, Canguilhem) mais les conditions de possibilité de certains discours qui sont des pratiques discursives. Qui plus est, ces conditions de possibilité ne sont pas des conditions kantiennes, mais des conditions d'existence pour des énoncés. Nous aurons l'occasion d'indiquer que, pour nous, l'archéologie foucaldienne est une épistémologie. (voir note 56). 
oblige à réinterpréter dans une dimension verticale ${ }^{36}$ la belle rectitude qui conduit la pensée rationnelle jusqu'à l'analyse de la folie comme une maladie mentale exclue par la société projet archéologique de l'Histoire de la folie - et le fait de reconnaître que "l'Histoire de la folie faisait une part beaucoup trop considérable, et d'ailleurs bien énigmatique, à ce qui s'y trouvait désigné comme une "expérience ", montrant par là combien on demeurait proche d'admettre un sujet anonyme et général de l'histoire ${ }^{37}$.

L'originalité du projet foucaldien réside en grande mesure dans le fait que l'on n'étudie pas un discours déjà fait, mais les conditions de sa production. Faire l'histoire de la folie c'est renoncer à la commodité des vérités définitives, c'est renoncer à tout repos dans la vérité et à la plénitude d'un savoir réalisé, terminé. Faire l'histoire de la folie c'est « s'affranchir de tout un jeu de notions qui diversifient, chacune à leur manière, le thème de la continuité ${ }^{38}$. C'est aussi, donc, dénoncer la fonction fondatrice du sujet. De même que dans la physique, l'économie, la philosophie ou la biologie, il n'est pas possible d'admettre l'unité d'un discours sur la folie. Telle unité est artificielle ; mieux, elle est arbitraire. Elle veut préserver les notions de tradition, permanence, influence, évolution, esprit etc., mais elle ne permet pas d'individualiser un ensemble d'énoncés et de les mettre en relation. Ainsi, loin de se fonder sur le "référent » folie, qui n'est autre que l'illusion que le présent se forge de son passé, l'archéologie cherche à formuler la loi de répartition d'un ensemble d'énoncés, la « loi de dispersion de différents objets ou référents mis en jeu par un ensemble d'énoncés, dont l'unité se trouve précisément définie par cette loi ${ }^{39}$. Foucault donne à ceci le nom de référentiel. Traditionnellement, tout arrive comme si la psychopathologie se référait à un seul objet formé une fois pour toutes et que l'histoire se serait chargée de transporter plus ou moins

36. Historre de la folie, p. 40. Sur le thème de la dimension verticale de l'analyse, voir $L$ 'archéologze du savoir, p. 96,115 et 143 ; la préface de 1961 de l'Historre de la folie, p. III et La naissance de la clinique, préface.

37. M. Foucault, L'archéologie du savoir, p. 26-27.

38. Ibid., p. 31.

39. M. Foucault, Réponse au cercle d'éptstémologie, p. 23 ; voir aussi L'archéologie du savoir, p. 5567. 
tranquillement jusqu'à son présent idéal. Or les mutations profondes que l'histoire a souffertes depuis plusieurs décennies montrent que " le problème n'est plus de la tradition et de la trace, mais de la découpe et de la limite ; ce n'est pas celui du fondement qui se perpétue, c'est celui des transformations qui valent comme fondation et renouvellement des fondations $"{ }^{40}$. On ne peut pas partir de la science constituée - la psychopathologie - comme si la folie était une entité dont les contours pouvaient être délimités par l'analyse rétrospective. Il faut recourir, en dehors de toute intention prescriptive, à l'espace dans lequel surgissent, se distribuent et se transforment les objets. Contre la règle cartésienne de la simplicité, il est nécessaire de renvoyer les énoncés à la complexité structurale de l'espace où ils vivent. Citons Foucault : "L'unité des discours sur la folie ne serait pas fondée sur l'existence de l'objet «folie », ou la constitution d'un horizon unique d'objectivité ; ce serait le jeu des règles qui rendent possible pendant une période donnée l'apparition d'objets : objets qui sont découpés par des mesures de discrimination et de répression, objets qui se différencient dans la pratique quotidienne, dans la jurisprudence, dans la casuistique religieuse, dans le diagnostique des médecins, objets qui se manifestent dans des descriptions pathologiques, objets qui sont cernés par des codes ou recettes de médication, de traitement, de soins. En outre, l'unité des discours sur la folie, ce serait le jeu des règles qui définissent les transformations de ces différents objets, leur non-identité à travers le temps, la rupture qui se produit en eux, la discontinuité interne qui suspend leur permanence. D'une façon paradoxale, définir un ensemble d'énoncés dans ce qu'il a d'individuel consisterait à décrire la dispersion de ces objets, saisir tous les interstices qui les séparent, mesurer les distances qui règnent entre eux, en d'autres termes formuler leur loi de répartition ${ }^{41}$. Tout ce projet méthodologique se maintient dans ce que Foucault appelle « une espèce de relativité ». Il ne s'agit toutefois pas, comme déjà l'indiquait Maladie mentale et personnalité, de culturalisme ${ }^{42}$. M. Foucault va au-delà, à la

\footnotetext{
40. M. Foucault, L'àrchéologie du savoir, p. 12 ; notons la référence critique aux traces derridiennes.

41. Ibid., p. 46-47.

42. Sur le relativisme de Foucault, voir M. Amiot : "Le relativisme culturaliste de Michel Foucault ", Les temps modernes, no 248, janvier 1967, p. 1271-1298.
} 
recherche d'un au-delà — ou d'un en-deçà — du donné phénoménologique, d'une "structure " qui est de l'ordre de l'impensé. Et son interrogation englobe jusqu'à la perspective culturaliste elle-même qui tend à établir l'adéquation folie-déviation en conservant une vision négative de la folie. Or il existe aussi un sens positif de la folie : la folie prend un sens à partir de ce qui la rejette et la dénonce comme folie, et c'est ce sens qui nous permet de comprendre en quoi consiste le double projet de Foucault. La perception que l'homme moderne a de son temps et de son espace fait apparaître une structure de rejet, c'est-à-dire de répression. La synchronie temporelle et structurale " grand renfermement " - «éditations métaphysiques » rend compte du geste qui tait la folie, et c'est à partir de lui qu'à la fin du

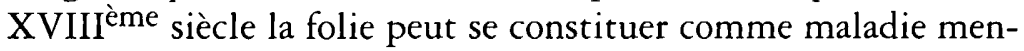
tale, comme objet scientifique. Mais, « est constitutif le geste qui partage la folie, et non la science qui s'établit, ce partage une fois fait, dans le calme revenu. Est originaire la césure qui établit la distance entre raison et non-raison ; . . . ${ }^{43}$. Parce qu'elle est un monologue de la raison sur la folie, la psychiatrie ne peut rien nous dire sur ce "degré zéro » de l'histoire de la folie, sur ce moment où la folie est expérience non encore partagée du partage lui-même. Au mieux, la psychiatrie ne nous donnera qu'une définition négative de la folie. Or, avant la séparation, c'est-à-dire rappelons-le, avant Descartes, il y eut une sorte d'état sauvage pendant lequel des expériences encore non séparées communiquaient entre elles par le biais du langage. Le projet de Foucault est donc double. Il s'agit, d'une part, de faire l'histoire du coup de force par lequel les hommes, dans le geste de la raison souveraine, séparent raison et déraison. Il s'agit, d'autre part, de faire l'archéologie d'un silence, l'histoire des structures du tragique tuées par le coup de force qui divisa ce qui, avant la séparation, était encore une expérience indifférenciée. Face à ce double projet, il n'est guère difficile de voir que le soleil nietzschéen illumine la prétention foucaldienne de confronter les dialectiques de l'histoire aux structures immobiles du tragique. Et si Foucault privilégie l'étude de la séparation, de l'exclusion, c'est parce que « c'est seulement dans l'acte de la séparation et à partir de lui » qu'on peut penser l'expérience

43. M. Foucault, Histoire de la folie, (1961), préface, p. I-II. 
tragique de la folie ${ }^{44}$. Il est même loisible d'affirmer que la " nécessité de la folie » est liée à la " possibilité de l'histoire ". Faire une étude structurale du référentiel folie c'est faire l'histoire des notions, des mesures répressives, des institutions, des concepts scientifiques qui emprisonnent une folie originaire et son langage. Ce que, selon nous, veut dire Foucault c'est que la folie n'est pas seulement un objet de connaissance, mais aussi un moyen de connaissance. En d'autres termes, si la folie est aujourd'hui un objet scientifique c'est parce qu'elle eut - et surtout parce qu'elle a encore de nos jours - une valeur exemplaire d'expression. Toutefois, à défaut de restituer l'état sauvage de la folie, à défaut d'accécirr à cette pureté primitive, l'étude structurale du référentiel folie «doit tendre à découvrir l'échange perpétuel, l'obscure racine commune, l'affrontement originaire qui donne sens à l'unité aussi bien qu'à l'opposition du sens et de l'insensé ${ }^{45}$.

\section{3- RAISON ET FOLIE CHEZ DESCARTES.}

Nous voudrions maintenant montrer que loin d'être exclue par l'exercice de la ratio cartésienne, comme le prétend Foucault, la folie est une expérience que tout sujet pensant doit faire s'il veut mener à bien la construction d'un système fermé de pensée. Si Foucault a raison lorsqu'il affirme que la nécessité de la folie est intimement liée à la possibilité de l'histoire, il a tort lorsqu'il fait du cas Descartes un modèle. De fait, l'acte de décision qui unit et sépare raison et folie est beaucoup plus vieux que celui que Foucault ramène aux Méditations métaphysiques. "Ce geste de décision qui détache du bruit de fond et de sa monotonie continue un langage significatif qui se transmet et s'achève dans le temps ${ }^{46}$ n'est, en effet, pas propre à Descartes mais constitue l'authentique sédimentation archaïque de la pensée occidentale. La structure d'exclusion, le fait qu'une nouvelle forme de discours (logos) déplace, exclut et exile une forme antérieure est un invariant qui se répète tout au long de l'histoire de la philosophie, c'est-à-dire tout au long de l'histoire du sens, de l'histoire de la raison en général. Il s'agit alors de voir si cette struc-

44. Ibid., p. VII.

45. Loc. cit.

46. Ibid., p. VI. 
ture d'exclusion implique la disparition de la folie ou si, au niveau de l'histoire des systèmes de pensée, chaque forme nouvelle de discours n'est en fait que l'expression d'une détermination particulière de la raison qui a besoin de faire l'expérience de la folie. Anticipant quelque peu notre réponse, nous dirons que la raison n'exclut pas la déraison ; elle construit par voie de fiction son autre, elle crée son dehors pour le désarmer et faire siens les pouvoirs que de tout temps l'homme a su rencontrer dans la folie.

\subsection{Le problème de la folie grecque et les héros de la culture.}

Alors la déraison disparut ! Telle pourrait être la sentence qui annoncerait de façon solennelle l'ère de la positivité. Cette sentence n'exprimerait toutefois qu'un long et tortueux mouvement qui n'arrive jamais à effacer les figures de ce que Foucault appelle "l'expérience tragique " de la folie. La raison pour laquelle Foucault se refuse de traiter le problème de la folie grecque $^{47}$ s'explique par la lumière nietzschéenne qui éclaire la recherche archéologique ${ }^{48}$. Foucault, en effet, accepte cò̀mme allant de soi la présentation antithétique de l'élan apollinien et de l'élan dionysiaque et, en suivant la lecture qui voit dans la connaissance dionysiaque l'intuition de la douleur du monde et dans l'œuvre apollinienne le monde de l'art comme libération, il donne la prééminence à Dionysos quant à l'origine de la sagesse. Or il est nécessaire de modifier la lecture unilatérale de Nietzsche et de montrer qu'en Grèce la sagesse et la connaissance se manifestent par le biais de la parole (logos) et qu'à Delphes, là où la parole est prononcée, c'est Apollon et non pas Dionysos qui

47. Histoire de la folie, p. 200 : "Si on laisse de côté le grand problème de la folie grecque . . . "

48. Sur le choix par Foucault de l'époque classique, voir L'archéologie du savoir, p. 230-231 : « L'archéologie désarticule la synchronie des coupures, comme elle aurait disjoint l'unité abstraite du changement et de l'événement. L'époque n'est ni son unité de base, ni son horizon, ni son objet : si elle en parle, c'est toujours à propos de pratiques discursives déterminées et comme résultat de ses analyses. L'âge classique, qui fut souvent mentionné dans les analyses archéologiques, n'est pas une figure temporelle qui impose son unité et sa forme vide à tous les discours ; c'est le nom qu'on peut donner à un enchevêtrement de continuités et de discontinuités, de formations discursives qui apparaissent et qui disparaissent ". Selon J. Baudrillard, Oubleer Foucault, Paris, Éditions Galilée, 1977, p. 12-13, Foucault serait "Le dernier dinosaure " de L' " ère classique ", d'une époque qui " est en train de basculer entièrement". 
parle. Sans vouloir faire une étude détaillée de ce problème ${ }^{49}$, nous devons signaler rapidement comment le fait de laisser de côté le problème de la folie grecque pose un certain nombre de difficultés.

Peut-être aurions-nous pu accepter les raisons pour lesquelles Foucault privilégie l'époque classique dans son analyse de la folie s'il n'avait pas affirmé que le Logos grec n'avait pas de contraire : " les Grecs avaient rapport à quelque chose qu'ils appelaient úßpıs. Ce rapport n'était pas seulement de condamnation ; l'existence de Thrasymaque ou celle de Calliclès suffit à le montrer, même si leur discours nous est transmis, enveloppé déjà dans la dialectique rassurante de Socrate. Mais le Logos grec n'avait pas de contraire ${ }^{50}$. En réalité, si la dialectique socra-

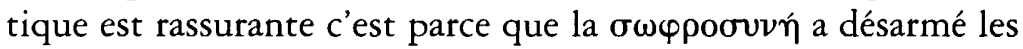
pouvoirs de l'úßpıs (l'excès, la démesure). "L'ignorance " socratique dénonce le vide d'idées du discours de la sophistique, et l'art du dialogue uni à l'épapogué s'achemine pas à pas vers une définition de la bonne rhétorique qui peut répondre aux inquiétudes de Gorgias : « . . si tu savais Socrate ! Si tu savais que d'une certaine façon la rhétorique a tous les pouvoirs entre ses mains ! ". S'il est vrai que la rhétorique - et la dialectique ont en commun de procéder par discours (dia logoi), la dialectique seule se soumet au Logos (le Bien), tandis que la rhétorique (empirie, savoir-faire) procède sans Logos (alogos), c'est-à-dire sans raison. Cette dernière appartient à la persuasion qui offre la croyance sans la science puisque "l'art oratoire n'a aucunement besoin d'avoir une connaissance profonde des choses; il lui suffit d'avoir trouvé un moyen de persuasion qui lui permet d'apparaître face aux ignorants comme plus savants que ceux qui sont vraiment savants ${ }^{51}$. Savoir formel qui ne requiert aucun objet déterminé, la rhétorique est le produit de l'expérience et de la routine : elle n'est qu'une mauvaise copie d'une partie de la politique, due à cette pernicieuse entreprise qui porte le nom générique de flatterie, et elle est la victime de l'immédiateté des désirs et des plaisirs (contre Pôlos et Calliclès). Or, déjà dans

49. Voir par exemple G. Colli, La nascita della filosofia, Milano, Adelphi edizioni, 1975 et Dopo Nietzche, Milano, Adelphi E, 1974.

50. Histoire de la folie, (1961), préface, p. III.

51. Platon, Gorgtas, 459c. 
l'Eutyphron, Socrate oppose au savoir formel de la sophistique la forme (eidos) à quoi tout ce qui est pieux doit de l'être et tel qu'en l'utilisant comme modèle $(\pi \alpha \rho \alpha \zeta \epsilon \iota \gamma \mu \alpha)$ on déclarera pieux ce qui sera tel que lui. Il existe ainsi, d'une part, la sophistique qui ne subordonne pas la vie au Logos (raison, mesure, harmonie), mais à une morale de l'instinct liée à l'exercice du pouvoir, qui procède sans Logos et a recours à l'art oratoire et, d'autre part, l'homme bon qui parle avec le regard posé sur une fin déterminée, la forme. Il s'agit donc de deux genres de vie dont le choix engage la vie sur un plan philosophique et ontologique. Seul le genre de vie philosophique mérite le titre d'Art, parce qu'il saisit la nature ( $\varphi v ́ \sigma \iota \varsigma)$ et la cause $(\alpha i \tau i \alpha)$ de son objet, en formant un Logos. Au contraire, le genre de vie "rhétorique » n'est qu'une routine $(\dot{\epsilon} \mu \pi \epsilon \iota \rho \hat{L} \alpha)$ qui procède sans Logos ( $\alpha \lambda \sigma \gamma \omega \varsigma)$. " En face du modèle idéal d'une âme où règnent arrangement et ordre, Socrate dresse le surhomme de Calliclès, aussi insociable

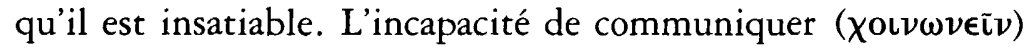
est due à ce qu'il s'est soustrait à la loi du cosmos et de l'être. En effet, ce qui joint ensemble Ciel et Terre, dieux et hommes, dans la communion, l'amitié, la justice, l'ordonnance, la discrétion, bref l'ordre, ce ne peut être ni l'ußpıs ou la $\pi \lambda \epsilon o \nu \epsilon \xi \zeta \alpha$ préconnisées par Gorgias ni la plate égalité souhaitée aveuglément par le vulgaire, c'est une sorte d'égalité, certes, mais l'égalité au sein même de l'inégalité, en deux mots : l'égalité géométrique $"$ " 2 .

Lire sous la plume de Foucault que « le Logos grec n'avait pas de contraire " est d'autant plus difficile à admettre, que 9 ans après sa préface de 1961, le même Foucault affirme dans sa Legon inaugurale au Collège de France : " chez les poètes grecs du $\mathrm{VI}^{\mathrm{e}}$ siècle encore, le discours vrai - au sens fort et valorisé du mot - le discours vrai pour lequel on avait respect et terreur, celui auquel il fallait bien se soumettre, parce qu'il régnait, c'était le discours prononcé par qui de droit et selon le rituel requis ; c'était le discours qui disait la justice et attribuait à chacun sa part ; c'était le discours qui, prophétisant l'avenir, non seulement annonçait ce qui allait se passer, mais contribuait à sa réalisation, emportait avec soi l'adhésion des hommes et se

52. P.B. Grenet, Histoire de la philosophie ancienne, Paris, Beauchesne, 1960, p. 90-91. 
tramait ainsi avec le destin. Or voilà qu'un siècle plus tard la vérité la plus haute ne résidait plus déjà dans ce qu'était le discours ou dans ce qu'il faisait, elle résidait en ce qu'il disait : un jour est venu où la vérité s'est déplacée de l'acte ritualisé, efficace, et juste, d'énonciation, vers l'énoncé lui-même : vers son sens, sa forme, son objet, son rapport à sa référence. Entre Hésiode et Platon un certain partage s'est établi, séparant le discours vrai et le discours faux ; partage nouveau puisque désormais le discours vrai n'est plus le discours précieux et désirable, puisque ce n'est plus le discours lié à l'exercice du pouvoir. Le Sophiste est chassé ${ }^{53}$. En effet, si Foucault parle de "la grande séparation platonicienne " comme l'un des moments de l'histoire des vérités coercitives, s'il parle de la dialectique rassurante de Socrate, comment peut-il alors sauver l'originalité du geste cartésien qui sépare raison et déraison? De fait, l'acte de décision qui unit et sépare raison et folie est beaucoup plus vieux que celui que Foucault veut voir dans les Méditations métaphysiques de Descartes. Le moment socratique marque la victoire de la dialectique sur l'húbris de Trasymaque et Calliclès et, donc, la victoire d'un nouveau système de discours qui exclut, déplace et exile un autre système. Telle structure d'exclusion n'est pas, par conséquent, propre à Descartes, mais est un invariant qui court tout au long de l'histoire de la philosophie et/ou de la raison. C'est seulement au prix de cette rectification que, croyonsnous, l'idée foucaldienne selon laquelle la nécessité de la folie est liée à la possibilité de l'histoire prend tout son sens. Il semble cependant que Foucault ne s'intéresse pas à l'histoire des hommes anonymes, mais à celle des héros de la culture. Il semble que la volonté de vérité, cette prodigieuse machine conçue pour exclure, trouve une sorte de justification négative, de raison d'être inespérée, dans les intuitions de la douleur du monde (connaissance nietzschéenne-dionysiaque) qu'eurent certaines figures privilégiées : "Tous ceux qui, écrit Foucault, de point en point dans notre histoire, ont essayé de contourner cette volonté de vérité

53. M. Foucault, L'ordre du discours, op. cit., p. 16-18. Cette volonté de vérité est un système d'exclusion, voir ibid, p. 19. Un peu plus lon, se proposant le projet de poursuivre l'analyse des systèmes d'exclusion Foucault écrit : « . . . je me placerai d'abord à l'époque de la sophistique et de son début avec Socrate ou du moins avec la philosophie platonicienne, pour voir comment le discours efficace, le discours rituel, le discours chargé de pouvoirs et de périls s'est ordonné peu à peu à un partage entre discours vrai et discours faux ", p. 64. 
et de la remettre en question contre la vérité, là justement où la vérité entreprend de justifier l'interdit et de définir la folie, tous ceux-là, de Nietzsche, à Artaud et à Bataille, doivent maintenant nous servir de signes, hautains sans doute, pour le travail de tous les jours ${ }^{54}$. Or c'est précisément dans cette idée qui apparaît comme un leitmotiv ${ }^{55}$ que nous croyons voir le fondement éthique (le privilège donné à une expérience de la douleur comme source de la connaissance) de l'Histoire de la folie. C'est elle que vise la méthode archéologique de monstration ${ }^{56}$ qui veut atteindre dans l'histoire (?) ce « degré zéro » de l'histoire, lorsqu'existait une expérience indifférenciée de la folie, lorsqu'il y avait communication entre ce qui n'était pas encore séparé, c'està-dire avant que la folie ne soit enfermée par la raison. Mais, l'impossibilité de réaliser cette tâche — impossibilité, puisque cela impliquerait reconstruire ce qui n'a pas de temporalité, parler de l'ineffable, parler de la folie qui est « absence d'œuvre » et parler depuis la raison qui a tu et domestiqué cette expérience - conduit Foucault à affirmer que « c'est seulement dans l'acte de la séparation et à partir de lui » qu'on peut penser « l'expérience tragique » de la folie.

54. Ibid., p. 22-23.

55. Histoire de la folie. p. 181-182; 187-188; $364 ; 380$ et suiv ; $383 ; 397$ et suiv ; 530 et 549 et suiv.

56. Se référant au discours de Foucault, Baudrillard écrit : « . . . ce n'est donc pas un discours de vérité, mais un discours mythique, au sens fort du terme, et je cross secrètement sans illusion sur l'effet de vérité qu'il produit. C'est d'allleurs ce qui manque à ceux qui, suivant les traces de Foucault, passent à côté de cet agencement mythique et se retrouvent avec la vérité, rien que la vérité ", in Oublier Foucault, op. cit., p. 11-12. À ce judicieux commentaire il faudrait ajouter que Foucault lui-même est de ceux qui " passent à côté de cet agencement mythique ". Foucault, en effet, prétend produire le réel dans le sens de rendre visible, de faire apparaître et comparaître (pro-ducere) ce qui est ; il s'agit pour lui d'une monstratton, c'est-à-dire d'une opération qui vise à connaître sañs intention prescriptive. Mais, pour le problème qui nous occupe, avec quel langage peut-on faire l'histoire de la séparation (ou de quelqu'histoire que ce soit, puisque toute histoire est histoire de la raison) et l'archéologie d'un silence, si ce n'est avec le langage de la raison. De là que la monstration foucaldienne est une dé-monstration. En plus du fait que la monstration ne peut se passer de son champ éthique de valorisation, Foucault démontre lorsqu'il indique larticulation interne des discours. Voir notre note 35 . Signalons enfin la conclusion de l'article que R. Barthes dédie à l'Histoire de la folie, : "l'histoire de la folie ne pourrait être "vraie " que si elle était naïve, c'est-à-dire écrite par un fou ; mais elle ne saurait alors être écrite en termes d'histoire, et nous voicı renvoyés à la mauvaise foi incoercible du savoir. C'est là une fatalité qui dépasse de beaucoup les simples rapports de la folie et de la déraison ; en fait, elle frappe toute " pensée ", ou pour être plus exact, tout recours à un méta-langage, quel qu'il soit : chaque fois que les hommes parlent $d u$ monde, ils entrent au cœur du rapport d'exclusion, lors même qu'ils parlent pour le dénoncer : le méta-langage est toujours terroriste . . " , in Savour et folie, Paris, Critique No 174, 1961 , p. 922. 


\subsection{Le « cas» Descartes.}

Où réside l'erreur des jugements que portent les sujets humains? Dans les sens ? Dans la déraison? Dans les songes, ou dans la séquence entrelacée des choses ? Telles sont quelquesunes des alternatives qu'offre la première méditation de Descartes et sur lesquelles nous allons maintenant nous appuyer pour interpréter la triple relation raison - folie - histoire. Lisant Descartes, nous croyons voir que c'est lorsque le sujet pensant fait rationnellement l'essai irrationnel de penser sans Dieu (le pouvoir-douter) et de fonder depuis lui-même ce qu'il n'est pas, qu'il découvre qu'il dépend de cet autre qui n'est pas lui. Mais, si c'est la vérification de cette impossibilité de fonder l'autre (Dieu, l'infini) depuis le sujet pensant qui fait apparâttre Dieu comme l'autre de la subjectivité finie, il faut voir dans la fiction du Dieu trompeur l'argument-clé de cette vérification. Loin d'exclure la folie, Descartes porte donc la fiction jusqu'à ses dernières conséquences, le Cogito fou et la folie universelle, parce qu'il sait que sans Dieu la pensée finie n'a pas le droit d'exclure la folie. Si elle l'exclut, ce sera de fait, violemment, dans l'histoire, de sorte que la folie est intimement liée à la possibilité de l'histoire. Nous connaissons la lecture foucaldienne ; il y a une synchronie temporelle et structurale entre le " grand renfermement " de la déraison et les Méditations métaphysiques de Descartes. Internement social de la folie et internement métaphysique de la déraison sont les effets d'un même geste d'exclusion car "si l'bomme peut toujours être fou, la pensée, en tant qu'exercice de la souveraineté d'un sujet qui se considère avec le devoir de percevoir le vrai, ne peut pas être fou ». Dans des paragraphes de grande profondeur, Foucault reconnaît cependant que le "coup de force " cartésien non seulement n'a pas pu effacer les valeurs dramatiques de la folie, mais que Descartes luimême n'a pas pu se libérer de la déraison. Rappelant grâce à quelle décision Descartes évitait dans la marche du doute la possibilité d'être fou, Foucault écrit : «...l'homme du XVII ${ }^{\mathrm{e}}$ siècle découvre, dans l'immédiate présence de sa pensée à elle-même, la certitude dans laquelle s'énonce la raison sous sa forme première. Mais cela ne veut pas dire que l'homme classique était, dans son expérience de la vérité, plus éloigné de la déraison que 
nous pouvons l'être nous-mêmes. Il est vrai que le Cogito est commencement absolu ; mais il ne faut pas oublier que le malin génie lui est antérieur. Et le malin génie n'est pas le symbole dans lequel sont résumés et portés au système tous les dangers de ces événements psychologiques que sont les images des rêves et les erreurs des sens. Entre Dieu et l'homme, le malin génie a un sens absolu : il est dans toute sa rigueur la possibilité de la déraison et la totalité de ses pouvoirs. Il est plus que la réfraction de la finitude humaine ; il désigne le péril qui, bien audelà de l'homme, pourrait l'empêcher de manière définitive d'accéder à la vérité : l'obstacle majeur, non de tel esprit, mais de telle raison. Et ce n'est pas parce que la vérité qui prend dans le Cogito son illumination finit par masquer entièrement l'ombre du malin génie, qu'on doit oublier son pouvoir menaçant : jusqu'à l'existence et à la vérité du monde extérieur ce danger surplombera le cheminement de Descartes ${ }^{57}$. Les idées qui peuvent être sauvées dans cette longue citation sont nombreuses. Toutefois, prise dans son détail l'interprétation s'avère erronée. Voyons pourquoi.

1) Certes, le Cogito est la première certitude de la raison et est un commencement absolu. Mais Foucault oublie que le Cogito est aussi une exception ${ }^{58}$. Cette première certitude, en effet, ne met pas fin au doute universel, comme le montre le fait que l'hypothèse du Dieu trompeur est maintenue dans la troisième méditation et cela, même si « la raison de douter qui dépend seulement de cette opinion, est bien légère, et pour ainsi dire métaphysique ». Le Cogito ne fait qu'offrir le critère de l'évidence propre à une vérité indubitable, mais à une vérité qui ne concerne que le « je » et non pas ce qui l'entoure. De fait, le doute se maintient jusqu'à la démonstration de "l'existence et la vérité du monde », mais telle existence et telle vérité à leur tour dépendent de la démonstration de l'existence de Dieu, car seulement alors peut se vérifier que ce qui est pour le « je " est aussi en soi : «... à présent je ne m'en (l'existence de Dieu) tiens pas seulement aussi assuré que de tout ce qui me semble le plus certain : mais, outre cela, je remarque que la certitude

57. Histoire de la folie, p. 175.

58. M. Guéroult, Descartes selon l'ordre des raisons, Paris, Aubier, 1953, vol. I, p. 50 et suiv. ; vol. II, p. 154 et suiv. 
de toutes les autres choses en dépend si absolument, que sans cette connaissance il est impossible de pouvoir jamais rien savoir parfaitement " $"$. L'erreur de Foucault réside dans sa confusion de la certitude de facto (le Cogito comme exception, le pour moi) et la certitude de jure (le Cogito et le monde fondés par les preuves de l'existence de Dieu, l'en soi).

2) Foucault se trompe lorsqu'il affirme que le malin génie est antérieur au Cogito, d'abord parce qu'il ne distingue pas Dieu trompeur et malin génie, ensuite parce qu'il assimile la folie au malin génie. On se souviendra que dans le premier appendice de l'bistoire de la folie — « Mon corps, ce papier, ce feu »- Foucault propose une double lecture des Méditations grâce à laquelle il prétend montrer qu'au niveau de l'exercice du sujet qui médite il y a une exclusion de l'argument de la folie, c'est-à-dire une exclusion d'une des propositions, " je ne serais pas moins extravagant, si je me réglais sur leurs exemples ", qui font partie du système. Fidèle à un certain structuralisme, Foucault croit que le système - niveau discursif, niveau démonstratif-logique de l'enchaînement des énoncés précède et détermine le sujet, à savoir que la folie (ici identifiée au malin génie) est antérieure au sujet. En réalité seule la distinction très problématique que Descartes opère entre l'analyse et la synthèse pourrait autoriser l'opposition catégorique entre système et exercice ou ascèse. Et, de fait, comme pour beaucoup d'exégètes de Descartes, le système-synthèse serait selon Foucault l'ordre des choses (ordo rerum) et l'exercice-analyse l'ordre de la connaissance (ordo cognitionis). Or rien ne permet une telle lecture ; système-synthèse et exercice-analyse sont tous deux, à leur façon, un ordre de connaissance. Cédons la parole à $\mathrm{F}$. Alquié : «je ne vois nulle part que l'ordre synthétique soit l'ordre des choses . . . La chose est vraiment l'unité ; c'est l'être, c'est l'unité confuse : c'est moi qui mets un ordre lorsque je connais. Et ce qu'il faut établir, c'est que l'ordre de ma connaissance, et qui est toujours un ordre de connaissance, qu'il soit synthétique ou analytique, est vrai. Car il me semble que tout ordre est lié à la connaissance, mais répond à la chose ${ }^{60}$.

59. R. Descartes, Cinquième méditation.

60. F. Alquié, Descartes, Cahiers de Royaumont, Paris, Minuit, 1957, p. 125. Voir aussi son édition des Oeuvres philosophiques de Descartes, vol. II, p. 583. Les commentaires d'Alquié 
Ainsi, que la folie n'appartienne pas au système ne veut pas dire que le sujet l'exclut pour pouvoir penser. Car si Foucault avait tenu compte de la distinction que $\mathrm{H}$. Gouhier établit entre le Dieu trompeur et le malin génie ${ }^{61}$, il se serait aperçu que, dans la mesure où il participe du mouvement hyperbolique de disqualification $d u$ fondement sensible de notre connaissance, l'argument du Dieu trompeur est une fiction ${ }^{62}$ qui est absolument nécessaire aux desseins de Descartes, savoir la substitution de l'axe aristotélico-thomiste de la connaissance qui va de l'être au connâitre par un axe qui va du connaître à l'être. Dans la marche du doute, le malin génie n'est donc pas la folie, mais un recours méthodologique qui permet au sujet qui veut établir « quelque chose de ferme et de constant dans les sciences » de persévérer dans sa défiance de la connaissance qu'offrent les sens. En maintenant l'actualité du doute, le malin génie permet de s'enfoncer encore plus dans la fiction de la tromperie divine, de la folie divine.

3) Une autre erreur de Foucault consiste à croire que le $\mathrm{XVII}{ }^{\mathrm{e}}$ siècle signifie une mise en relief univoque du sujet conscient de soi. Notre thèse est que ce n'est pas le sujet pensant cartésien qui exclut la folie, mais Dieu. La folie est la compagne de la pensée aussi longtemps que Dieu ne garantit pas que le monde est rationnel. L'exercice du doute dont le but est, rappelons-le, de

sont dirigés contre l'interprétation de M. Guéroult, op. cit., vol. I, p. 25 et suiv., pour qui l'ordre synthétique serait l'ordre de l'existence réelle, l'ordre de la dépendance en soi des réalités.

Le lecteur pourra consulter l'article « Mais quoi ce sont des fous " où, " pour donner un statut acceptable à la folie ", J-M Beyssade invite à " chercher dans quelle mesure ce qui suit peut réagir sur ce qui précède, c'est-à-dire revenir sur ce que Descartes a appelé l'ordre des raisons, sur le dogme qui a conduit Guéroult à d'admirables analyses, mais aussi à quelques conséquences téméraires, que la plupart des commentateurs ont autant de peine à admettre que les conclusions de $\mathrm{M}$. Foucault ", in Revue de métaphysique et de morale, Paris, Juillet-Septembre 1973, p. 292. Selon lui, l'ordre des raisons est subordonné à une règle inverse, celle de la cobérence, "où ce qui précède dépend, dans son sens et dans son intention, de ce qui vient ensuite ", ibid., p. 293. C'est cette règle qui explique comment la folie peut être convoquée puis congédiée "sans enfermer le sujet méditant dans une immuable qualification".

61. H. Gouhier, Essais sur Descartes, Paris, Vrin, 1973, p. 162 et suiv.

62. Se référant à l'hypothèse du Dieu trompeur, Descartes écrit dans la première méditation : «... supposons ... que tout ce qui est dit ici d'un Dieu soit une fable (fictitium)". "C'est pourquoi je pense que j'en (l'hypothèse du Dieu trompeur) userai plus prudemment, si, prenant un parti contraire, j'emploie toutes mes forces à me tromper moi-même, feignant (fingam) que toutes ces pensées sont fausses et imaginaire ». Descartes corrobore dans plusieurs textes le fait que la tromperie divine est une fiction; voir, par exemple l'Entretien avec Burman et la lettre à Buitendjick de 1643. 
disqualifier le fondement sensible de notre connaissance, doit être compris depuis la problématique de la démonstration de l'existence de Dieu, puisque seule celle-ci peut fonder ma certitude. Or le fait de douter est déjà un indice de l'existence de Dieu dans la mesure où il signale la présence en moi de l'infini : «... j'ai, écrit Descartes, en quelque façon premièrement en moi la notion de l'infini, que du fini, c'est-à-dire de Dieu, que de moi-même. Car comment serait-il possible que je puisse connaître que je doute et que je désire, c'est-à-dire qu'il me manque quelque chose et que je ne suis pas tout parfait, si je n'avais en moi aucune idée d'un être plus parfait que le mien, par la comparaison duquel je connaîtrais les défauts de ma nature ? ${ }^{63}$. Mais, qui plus est nous pensons que l'hypothèse du Dieu trompeur constitue une sorte de première preuve de l'existence de Dieu pour les raisons suivantes : premièrement, parce que l'opposition entre doute naturel et doute métaphysique préfigure l'affirmation selon laquelle l'hypothèse du Dieu trompeur est une " opinion » et que la raison de douter qui en dépend " est bien légère, et pour ainsi dire métaphysique ». Deuxièmement, parce que dans la mesure où « du connâitre à l'être la conséquence est bonne " ${ }^{64}$, Descartes est amené à dire que l'idée de Dieu est innée et, donc, à découvrir dans la finitude du sujet pensant l'infinité de Dieu. De là la nécessité d'éliminer toute possibilité d'une manifestation subjective de la folie qui pourrait faire de l'idée que l'on a une exception, une extravagance. Troisièmement, parce qu'il est nécessaire de supprimer l'irrationnel de l'univers ; à quoi servirait-il, en effet, de prouver l'existence de Dieu si l'on n'excluait pas auparavant toute manifestation d'irrationnalité dans le monde ? C'est pour cette raison, pensonsnous, que l'argument du Dieu trompeur, en tant qu'il est un appel à la folie universelle, met en évidence cette nécessité, car si la folie est "exclue " de la pensée c'est parce que la présence de la déraison - déraison entendue comme le produit de l'imagination qui est " une façon de penser particulière pour les choses matérielles " ${ }^{65}$ et qui nous reconduit vers l'enfance (l'aristotélico-thomisme) - pourrait continuer à menacer de renverser les fondements intellectuels de la connaissance. Il est donc correct

63. Troisième méditation. Voir aussi la lettre à Mesland du 2 avril 1644.

64. R. Descartes, Réponses aux septièmes objections.

65. R. Descartes, Discours de la méthode, quatrième partie. 
d'affirmer avec $\mathrm{O}$. Hamelin que « le malin génie n'est pas autre chose qu'une personnification de la violence que fait peut-être subir à l'esprit la nature peut-être irrationnelle de l'univers " ${ }^{66}$. Si le monde au milieu duquel nous sommes situés n'est pas rationnel, la pensée peut être dominée par des circonstances extérieures qui dictent nos jugements auxquels, dès lors, il ne reste plus qu'une apparence de rationalité qui ne les empêche pas d'être au fond irrationnels. La reconnaissance de l'identité de la pensée et de l'être ne s'atteint, il est vrai, que sur la base de l'exclusion de l'irrationnel sous ses différentes formes. Mais cette exclusion, d'une part dépend d'une preuve implicite de l'existence de Dieu, si par Dieu l'on comprend non seulement la présence d'un ordre dans le monde, mais aussi l'omnipotence qui rend impossible la tromperie, puisque celle-ci est un " défaut " et, d'autre part implique que pour mener à bien la méthode du doute il faut déjà être raisonnable. Ainsi, ce qui pour Foucault signifie l'exclusion de la folie par un sujet qui ne peut penser et être fou en même temps, est pour nous l'inclusion de la folie dans l'économie du doute cartésien . . . ou non-cartésien. Car s'il n'en était pas ainsi, la folie pourrait toujours menacer le sujet pensant et ce qui fonde son activité. En persistant comme sédimentation archaïque, la possibilité de la déraison n'offrirait alors qu'une probabilité de connaissance ${ }^{67}$. Mais surtout, si le sujet qui pense avait des doutes quant à sa propre rationalité, il serait naturellement conduit à suspecter tout raisonnement au moyen duquel il pourrait essayer d'établir son bon sens ${ }^{68}$. Descartes n'exclut

66. O. Hamelin, Le système de Descartes, Paris, Alcan, 1911, p. 118. Évidemment, malin génie doit être ici remplacé par hypothèse du Dieu trompeur. Pour la critique de l'interprétation d'Hamelin, voir H. Gouhier, op. cit., p. 168 et suiv.

67. Jusqu'à ce que nous n'ayons pas démontré que Dieu existe, c'est-à-dire que Dieu ne peut pas nous tromper, nous n'aurons pas " une véritable science, mais une simple persuasion... ", lettre à Regius du 24 mai 1640. Or le passage de la persuasion à la science n'est possible que si l'on épuise les raisons que l'on a de douter.

68. En critiquant les opinions que jusqu'alors il avait en sa créance, Descartes veut déterminer si les motifs de son doute sont ou non raisonnables. S'il commençait par mettre en question le fait qu'il est un homme, il ne pourrait pas mener à bon terme la tâche qu'il s'est fixée, savoir «établir quelque chose de ferme et de constant dans les sciences". Comme l'écrit H.G. Frankfurt, « the task he sets for himself in the Méditations is not to discover how a madman can find a foundation for the sciences. A madman cannot do so at all. Unless Descartes supposes himself to be sane he cannot conduct the investigation to which he wishes to devote himself. The question he poses as he undertakes his inquiry, therefore, is this : Assuming that I am a reasonnable man, how (if at all) can I attain certainty ? His project may fail, and he may for this reason ultimately be led to wonder whether he is not after all incapable of rational discriminations among judgments . . . Because Des- 
pas la folie afin de pouvoir penser ; il l'intègre à l'exercice même de la ratio comme une objection que la raison se fait à ellemême. De fait, toute critique de la raison est une critique que la raison s'adresse à elle-même.

Au moyen de l'exercice du doute Descartes forme rationnellement le projet irrationnel de penser sans Dieu. Il en arrive à se figurer (fingere) un Dieu qui trompe les hommes. Mais, au moment où le pouvoir-douter indique la puissance de la pensée, à savoir le fait que la pensée, raisonnable ou non, résiste aux attaques du malin génie, l'homme découvre que pour maintenir son actualité face à la succession discontinue des instants la pensée a besoin de Dieu pour garantir sa certitude, c'est-à-dire son exercice temporel. C'est donc par le biais du projet irrationnel d'une pensée capable de forger la fiction d'un Dieu trompeur que je me découvre fini. Et ce pouvoir-douter que Descartes avait initialement défini comme le pouvoir du sujet pensant apparaît maintenant comme le signe de la finitude humaine. La contradiction n'est toutefois qu'apparente. Voyons pourquoi. En privilégiant le système sur l'exercice Foucault s'interrogeait sur le sens de la métaphysique cartésienne depuis la perspective du sujet. L'exercice de la ratio qui décide de méditer implique certains choix - et, par conséquent certaines exclusions - si elle veut maintenir son actualité de sujet pensant. Pour nous, en revanche, le pouvoir-douter montre qu'il faut s'interroger sur le sens de la métaphysique cartésienne depuis la perspective de Dieu car la métaphysique cartésienne ne signifie nullement une mise en relief univoque du « je » conscient de soi, sinon que Dieu y est posé comme le vrai sujet qui détermine et soutient le monde dans sa totalité, y compris l'homme ${ }^{69}$. La loi de la métaphysique cartésienne consiste à appréhender la subjectivité et à conserver sa tension interne avec la transcendance. C'est lorsque je découvre l'infini en moi que je sais que je suis un être fini, et c'est ici que le doute méthodique acquiert toute sa

cartes' assumption is provisional and heuristic, it does leave the question of his own sanity open. But he cannot raise this question at the start of his inquiry without incoherently undermining the basis of his enterprise", Demons, dreamers and madmen. The defense of reason in Descartes's Meditations, Indianapolis, New York, Bobbs-Merrill, 1970, p. 38 ; voir aussi p. 82-84.

69. Voir. W. Schultz, El dios de la metafísica moderna, Mexico, F.C.E., 1961. 
dimension. De signe de la puissance de la pensée humaine, le pouvoir-douter se transforme en un chemin méthodologique nécessaire qui nous permet de comprendre que l'homme ne peut pas exister sans Dieu ou, en d'autres termes, qu'en tant que res cogitans l'homme doit présupposer Dieu, c'est-à-dire un ordre rationnel dans le monde. Si l'on assiste au début de la métaphysique moderne à une tendance à placer l'homme au premier plan, ce n'est certes pas pour diminuer la puissance de Dieu, mais plutôt pour mettre en évidence qu'au-dessus de cette subjectivité, il existe un Dieu philosophique qui limite et mesure l'exercice de la pensée. Car, s'il est vrai que la subjectivité humaine est la condition de la connaissance des êtres mondains, celle-ci est épistémologiquement impuissante vis-à-vis d'elle-même. Foucault a raison lorsqu'il insiste sur l'importance décisive de l'exercice du doute ; Descartes doit maintenir l'actualité du doute. Mais nous venons de voir que c'est précisément par le biais de ce pouvoirdouter que l'homme comprend qu'il est fini et découvre qu'il a l'idée de l'infini. En passant de la découverte de la res cogitans (quod) à la question de la nature (quid) de la res cogitans Descartes écrit : "Je suis, j'existe : cela est certain ; mais combien de temps ? À savoir, autant de temps que je pense ; car peut-être se pourraitil faire, si je cessais de penser, que je cesserais en même temps d'être ou d'exister ${ }^{70}$. Nous voici donc confronté à un problème fondamental, la relation entre le temps et la vérité, qui nous amène à nous interroger sur le critère d'évidence, le critère de vérité, à partir de l'hypothèse du Dieu trompeur.

Le fait de rencontrer de nouveau l'hypothèse du Dieu trompeur dans la troisième méditation est la preuve que ni le Cogito première certitude - ni la règle d'évidence - dont le Cogito est le modèle - ne sont des raisons suffisantes pour mettre un terme à la fiction de la tromperie universelle. Mais il y a plus : «la certitude de fait du Cogito lui-même constitue au sein du doute universel une exception aussi indiscutable en fait qu'injustifiée en droit. C'est pourquoi, dès que je me détache du Cogito actualisé pour l'objectiver par rapport à moi, en le situant dans l'ensemble des connaissances que mon entendement a toujours naturellement réputées pour vraies, je le retrouve confronté comme

70. Deuxième méditation. 
celles-ci avec l'hypothèse toujours valable du Malin Génie . . . Ainsi, malgré le fait, je suis obligé de revenir au doute, au nom du droit, c'est-à-dire au nom du principe de la tromperie universelle érigé depuis le début en règle imprescriptible de la recherche méthodologique $»^{71}$. En d'autres termes, la valeur du Cogito dépend de l'actualité de l'intuition qui le découvre. En effet, si le fait de penser implique mon existence - pour penser, il faut être - je ne suis que dans la mesure où j'ai une intuition. Or, étant donné la conception cartésienne du temps comme une succession discontinue d'instants ${ }^{72}$ rien ne me garantit qu'une intuition passée est vraie maintenant ou sera vraie demain : «... cette proposition : Je suis, J'existe, est nécessairement vraie, toutes les fois que je la prononce, ou que je la conçois en mon esprit ${ }^{73}$; la valeur du Cogito dépend de son actualité, c'est-àdire d'un instant dans le temps. L'importance du temps est telle que Descartes n'hésite pas à y recourir pour prouver l'existence de Dieu ${ }^{74}$. Mais l'importance du temps se juge tant par cette preuve a contingentia mundi que par le thème de la mémoire: puisque la certitude dépend d'un instant, celui de l'intuition présente, et puisque le temps est discontinu, le souvenir d'une certitude passée n'est une certitude maintenant que dans la mesure où Dieu en est le garant. De fait, la mémoire est une source d'erreur non seulement à cause des mécanismes psychologiques qui nous font oublier ou dénaturer les raisons et leur ordre, non seulement à cause du harcèlement continu des images sensibles qui peuvent nous renvoyer au cadre théorique aristotélico-thomiste, mais aussi à cause du principe méthodologique qui nous oblige à douter de tout ce qui est capté par l'intuition. Comme l'écrit Guéroult, " la certitude de la certitude, privilège du Cogito, est abolie dans le souvenir de ma certitude ${ }^{75}$. De même que les

71. M. Guéroult, op. cit., vol. I, p. 155

72. Voir Principes de la philosophie, I, 21.

73. Deuxième méditation.

74. "tout le temps de ma vie, écrit Descartes, peut être divisé en une infinité de parties, chacune desquelles ne dépend en aucune façon des autres; et ainsi, de ce qu'un peu auparavant j'ai été, il ne s'ensuit pas que je doive maintenant être, si ce n'est qu'en ce moment quelque cause me produise et me crée, pour ainsi dire, derechef, c'est-à-dire me conserve ". Selon Alquié, "Descartes use d'une preuve nouvelle parce que la précédente ne peut convaincre ceux qui, comme les thomistes (ou Descartes lui-même quand il relâche son attention), sont aveuglés par la connaissance sensible ", "Oeuvres philosophiques ", op. cit., vol. II, p. 448, note 3 .

75. Op. cit., vol. I, p. 157. 
autres connaissances, le Cogito souffre les assauts du doute ou, mieux, doit souffrir les assauts du doute à tel point que si le Cogito n'était pas une intuition présente, mais le simple souvenir d'une intuition passée, il serait un préjugé ${ }^{76}$. Nous comprenons maintenant pourquoi notre affirmation selon laquelle la subjectivité humaine est épistémologiquement impuissante vis-à-vis d'ellemême nous conduit vers la garantie que Dieu, et Dieu seul, offre à la mémoire ${ }^{77}$. C'est dans la temporalisation du Cogito, c'està-dire dans le chemin que la méthode parcourt depuis la découverte du Cogito jusqu'à la démonstration de l'existence de Dieu et de la garantie divine de la mémoire, qu'il faut voir non pas l'exclusion de la folie mais le mouvement qui intègre la fiction de la tromperie universelle - cogito, ergo sum ; dubito, ergo sum (critère de vérité) - à la certitude absolue que l'ordre de mes pensées est bien l'ordre des pensées - cogito, ergo sum ; Deus est, ergo non dubito (critère de véracité). Lorsque Descartes établit la dernière preuve de l'existence de Dieu, il peut alors se passer de la fiction de la folie du monde et aborder avec confiance et certitude son objectif fondamental, savoir démontrer " l'existence des choses matérielles et la réelle distinction entre l'âme et le corps de l'homme ". Lorsque Descartes finalement démontre l'existence de Dieu, le cogito échappe à la possibilité toujours présente de la folie car c'est Dieu qui garantit mes représentations et mes déterminations cognitives, c'est-à-dire mon discours contre la folie : "c'est Dieu qui exclut la folie et la crise, c'est-à-dire les « comprend » dans la présence résumant la trace et la différence. Ce qui revient à dire que la crise, l'anomalie, la négativité, etc., sont irréductibles dans l'expérience

76. Descartes, Lettre à Clerselier sur les cinquièmes objections.

77. Dieu est le seul fondement de la science : "Or, écrit Descartes, qu'un athée puisse connaître clairement que les trots angles d'un triangle sont égaux à deux droits, je ne le nie pas ; mais je maintiens seulement qu'il ne le connaît pas par une vraie et certaine science, parce que toute connaissance qui peut être rendue douteuse ne doit pas être appelée science ; et puisqu'on suppose que celui-là est un athée, il ne peut pas être certain de n'être point déçu dans les choses qui lui semblent être très évidentes, comme il a déjà été montré cidevant ; et encore que peut-être ce doute ne lui vienne point en la pensée, il lui peut néanmoins venir, s'il l'examine, ou s'il lui est proposé par un autre ; et jamais il ne sera hors du danger de l'avoir, si premièrement il ne reconnaît un Dieu ", Réponses aux secondes objections, "Oeuvres philosophiques", op. cit., vol. II, p. 565. Le savoir de L'athée reste sujet au doute métaphysique; il n'est donc pas à l'abri de toute objection (" . . . scientiam athei . . non esse immutabilem et certam . . ", V ve Resp., A.T., T. VII, p. 428, L. 1-9) et n'est par conséquent pas une vraie science. 
de la finitude ou d'un moment fini, d'une détermination de la raison absolue, ou de la raison en général. Vouloir le nier et prétendre assurer la positivité (du vrai, du sens, de la norme, etc.) hors de l'horizon de cette raison infinie (de la raison en général et au delà de ses déterminations), c'est vouloir effacer la négativité, oublier la finitude au moment même où l'on prétendait dénoncer comme une mystification le théologisme des grands rationalismes classiques . . . Mais Dieu, c'est l'autre nom de l'absolu de la raison elle-même, de la raison et du sens en général. Et qu'est-ce qui saurait exclure, réduire ou, ce qui revient au même, comprendre absolument la folie, sinon la raison en général, la raison absolue et sans détermination, dont l'autre nom est Dieu pour les rationalistes classiques ? ${ }^{78}$.

Le double signe du pouvoir-douter indique le désir cartésien de montrer à l'homme qui philosophe la finitude du sujet qui philosophe, du sujet qui revendique pour lui-même la possibilité et le droit de fonder depuis lui-même la certitude. Mais, ce que veut dire et ce que dit Descartes c'est que si l'usage de la raison se révèle être insensé dans son entreprise, cette déraison est absolument nécessaire. La folie en effet ne peut être exclue sans qu'elle ne soit préalablement convoquée par la raison, à l'intérieur de la pensée, dans le langage de la fiction, dans le langage de la folie.

Notre conclusion est que la force du rationalisme tient au fait qu'il montre les limites de la raison humaine par le biais d'un exercice fou de cette même raison. Mais, étant donné l'histoire de la pensée occidentale, nous pensons que cette conclusion peut s'appliquer à toute l'histoire de la philosophie. La nécessité de la folie est intimement liée à la possibilité de l'histoire. Nous avons dit plus haut que si le sujet qui pense avait des doutes quant à sa propre rationalité, il serait naturellement conduit à suspecter tout raisonnement au moyen duquel il pourrait essayer d'établir son bon sens. Un petit jeu de substitutions permet de mieux comprendre le sens de cette phrase si nous la lisons de la façon suivante : si le sujet qui pense avait des doutes quant à sa propre normalité, il serait naturellement conduit à suspecter

78. J. Derrida, op. cit., p. 89 , note $1-$ p. 90 , note 1 . 
tout raisonnement au moyen duquel il pourrait essayer d'établir qu'il est mentalement sain. En d'autres termes, tout discours, y compris celui des "fous", doit, s'il prétend être intelligible, porter en soi la normalité. Loin d'être exclue par une détermination historique de la pensée, la folie fonctionne donc dans l'histoire de la pensée comme un recours méthodologique une fiction qui permet de mettre des bornes à une pensée qui apparaît comme excessive vis-à-vis d'une autre pensée qui est dominante. Être fou c'est oser penser la différence à un moment où règne une pensée cohérente et suffisante du monde. Être fou c'est penser contre un système fermé de pensée . . . pour le remplacer par un autre système non moins cohérent et suffisant. Ainsi, contrairement à ce que croit Foucault, la tentative cartésienne de pénétrer dans l'expérience de la folie n'est pas une extravagance inacceptable pour un sujet qui veut méditer. Cette tentative est le signe que tout essai de la part du sujet de penser une réalité différente doit s'inscrire d'emblée dans la rationalité. Et le plus remarquable du « cas " Descartes c'est qu'il répète, à sa façon, l'acte constitutif de l'exercice de la pensée. Certes "le coup de force " cartésien qui privilégie la raison est une décision étbique, mais il n'est pas propre à Descartes car ce privilège est l'authentique sédimentation archaïque de la pensée. Être aujourd'hui cartésien c'est répéter l'acte qui, une fois, en un certain lieu, donna commencement à la pensée occidentale. L'expression être fou, expression qui n'a pas un sens philosophique univoque, ne dit alors que l'autre - cet autre de la subjectivité bumaine - de chaque forme déterminée du Logos roi. Être fou c'est affirmer, hier ou aujourd'hui, que le système fermé qui domine est nu ou qu'il n'est pas présent (absence que d'aucuns appellent de façon incorrecte idéologie) face au regard du sujet. Être fou c'est, hier avec Descartes, forger la fiction d'un Dieu trompeur ou, aujourd'hui faire sien "le rire qui secoue à sa lecture toutes les familiarités de la pensée. . ébranlant toutes les surfaces ordonnées et tous les plans qui assagissent pour nous le foisonnement des êtres, faisant vaciller et inquiétant pour longtemps notre pratique millénaire du Même et de l'Autre " ${ }^{79}$. Soyons donc fous, mais n'oublions pas que la folie n'est rien d'autre

79. M. Foucault, Les mots et les choses, Paris, Gallimard, 1966, p. 7. 
qu'une détermination historique de la raison. Et puis, comme disait Pascal, " les hommes sont si nécessairement fous que ce serait être fou par un autre tour de folie de n'être pas fou ".

Département de philosophie

Université d'Ottawa et Université de Cali (Colombie) 


\section{BIBLIOGRAPHIE}

M. Amiot, Le relativisme culturaliste de Michel Foucault, Les temps modernes, No. 248.

R. Barthes, Savoir et folie, Critique, no. 174.

J. Baudrillard, Oublier Foucault, Éditions Galilée.

J-M. Beyssade, "Mais quoi ce sont des fous », Revue de métaphysique et de morale, Juillet - Septembre 1973.

Cahiers de Royaumont, Descartes, ed, de Minuit.

G. Canguilhem, Mort de l'bomme ou épuisement du cogito ?, Critique, no 242.

P. Caruso, Conversazioni con claude Lévi-Strauss, Michel Foucault, Jacques Lacan, U. Mursia \& C.

G. Colli, La nascita della filosofia, Adelphi Edizioni. , Dopo Nietzsche, Adelphi Edizioni.

J. Derrida, L'écriture et la différence, Le seuil.

R. Descartes, Oeuvres philosophiques, Éd. F. Alquié, Garnier, 3 volumes.

Oeuvres de Descartes, publiées par C. Adam et P. Tannery, Vrin en co-édition avec le CNRS, XII volumes.

M. Foucault, Maladie mentale et personnalité, P.U.F.

$\longrightarrow$ Maladie mentale et psychologie, P.U.F.
Folie et déraison, Plon, 1961. Histoire de la folie à l'âge classique, Gallimard, 1972.

_. Histoire de la folie à l'âge classique, Tel, 1979.

— La naissance de la clinique, P.U.F. Les mots et les choses, Gallimard.

, Réponse au cercle d'épistémologie, Cahiers pour l'analyse no 9, Le seuil.

—, L'archéologie du savoir, Gallimard. L'ordre du discours, Gallimard.

H.G. Frankfurt, Demons, dreamers and madmen. The defense of reason in Descartes's Meditations, Bobbs-Merrill.

D. Giovannangeli, Écriture et répétition, 10/18.

H. Gouhier, Essais sur Descartes, Vrin.

P.B. Grenet, Histoire de la philosophie ancienne, Beauchesne.

M. Guéroult, Descartes selon l'ordre des raisons, Aubier, 2 volumes.

O. Hamelin, Le système de Descartes, Alcan.

S. le Bon, Un positiviste désespéré : M. Foucault, Les temps modernes, no 248. Platon, Gorgias.

A. Sheridan, Foucault. The will to truth, Tavistock publications.

W. Schultz, El Dios de la metafísica moderna, F.C.E.

E. Trias, Filosofía y carnaval, Anagrama. 